\title{
SINGULAR SETS FOR HARMONIC MEASURE ON LOCALLY FLAT DOMAINS WITH LOCALLY FINITE SURFACE MEASURE
}

\author{
JONAS AZZAM, MIHALIS MOURGOGLOU, AND XAVIER TOLSA
}

\begin{abstract}
A theorem of David and Jerison asserts that harmonic measure is absolutely continuous with respect to surface measure in NTA domains with Ahlfors regular boundaries. We prove that this fails in high dimensions if we relax the Ahlfors regularity assumption by showing that, for each $d>1$, there exists a Reifenberg flat domain $\Omega \subset \mathbb{R}^{d+1}$ with $\mathcal{H}^{d}(\partial \Omega)<\infty$ and a subset $E \subset \partial \Omega$ with positive harmonic measure yet zero $\mathcal{H}^{d}$-measure. In particular, this implies that a classical theorem of F. and M. Riesz fails in higher dimensions for this type of domains.
\end{abstract}

\section{CONTEnTs}

1. Introduction

2. The enlarged domain $\Omega_{\varepsilon}^{+}$

3. Radon measures of low dimension

4. Wolff snowflakes and harmonic measure 14

References

\section{INTRODUCTION}

The F. and M. Riesz theorem states that, for simply connected planar domains whose boundary has finite length, harmonic measure and arc-length are mutually absolutely continuous. The obvious generalization to higher dimensions is false due to examples of $\mathrm{Wu}$ and Ziemer: they construct topological two-spheres in $\mathbb{R}^{3}$ with boundaries of finite Hausdorff measure $\mathcal{H}^{2}$ where either harmonic measure is not absolutely continuous with respect to $\mathcal{H}^{2}[\mathrm{Wu}]$ or $\mathcal{H}^{2}$ is not absolutely continuous with respect to harmonic measure [Z], respectively. In spite of this, there has still been interest in narrowing down some sufficient conditions for when the F. and M. Riesz theorem still holds in higher dimensions. In fact, the study of the relationship

2010 Mathematics Subject Classification. 31A15,28A75,28A78.

Key words and phrases. Harmonic measure, absolute continuity, nontangentially accessible (NTA) domains, $A_{\infty}$-weights, doubling measures, porosity.

The three authors were supported by the ERC grant 320501 of the European Research Council (FP7/2007-2013). X.T. was also supported by 2014-SGR-75 (Catalonia), MTM2013-44304-P (Spain), and Marie Curie ITN MAnET (FP7-607647). 
between harmonic measure and the geometric and metric properties of domains is a very active area of research. See for example [AHMNT], [BL], [HMU] or [KPT].

Recall that a nontangentially accessible domain (or NTA domain) $\Omega \subset \mathbb{R}^{d+1}$ is a connected open set for which the following hold:

(1) $\Omega$ is a $C$-uniform domain, meaning for all $x, y \in \Omega$ there is $\gamma \subset \Omega$ for which $\mathcal{H}^{1}(\gamma) \leq C|x-y|$ and $\operatorname{dist}\left(z, \Omega^{c}\right) \geq \operatorname{dist}(z,\{x, y\}) / C$ for all $z \in \gamma$, and

(2) $\Omega$ satisfies the $C$-exterior corkscrew condition, meaning for all $\xi \in \partial \Omega$ and $r>0$ there is $B(z, r / C) \subset B(\xi, r) \backslash \Omega$.

In [DJ], David and Jerison show that if $\Omega \subset \mathbb{R}^{d+1}$ is an NTA domain and $\partial \Omega$ is Ahlfors regular, meaning there is $A>0$ so that

$$
r^{d} / A \leq \mathcal{H}^{d}(B(\xi, r) \cap \partial \Omega) \leq A r^{d} \text { for all } \xi \in \partial \Omega \text { and } r \in(0, \operatorname{diam} \Omega),
$$

then not only do we have $\left.\omega \ll \mathcal{H}^{d}\right|_{\partial \Omega} \ll \omega$, but they are in fact $A_{\infty}$-equivalent. At first look, Ahlfors regularity seems superfluous for establishing absolute continuity, and in some sense it is: in [Ba], Badger shows that if one merely assumes $\left.\mathcal{H}^{d}\right|_{\partial \Omega}$ is locally finite and $\Omega \subset \mathbb{R}^{d+1}$ is NTA, then we still have $\left.\mathcal{H}^{d}\right|_{\partial \Omega} \ll \omega$. He also shows that $\left.\omega \ll \mathcal{H}^{d}\right|_{\partial \Omega} \ll \omega$ on the set

$$
\left\{\xi \in \partial \Omega: \liminf _{r \rightarrow 0} \mathcal{H}^{d}(B(\xi, r) \cap \partial \Omega) / r^{d}<\infty\right\}
$$

but asks whether or not mutual absolute continuity holds on the whole boundary of $\Omega$ and not just on the above set (see Conjecture 1.3 in [Ba]). However, this turns out not to be true in general: we show that there exist domains with locally finite perimeter where $\omega$ is not absolutely continuous with respect to $\left.\mathcal{H}^{d}\right|_{\partial \Omega}$ even if we assume stronger conditions than the NTA property, such as Reifenberg flatness.

By a domain in $\mathbb{R}^{d+1}$ we mean an open connected set. Given $A, B \subset \mathbb{R}^{d+1}$, we denote by $\operatorname{dist}_{H}(A, B)$ the Hausdorff distance between $A$ and $B$.

Definition 1.1 (Reifenberg flat domain). Let $\Omega \subset \mathbb{R}^{d+1}$ be an open set, and let $0<\delta<1 / 2, r_{0}>0$. We say that $\Omega$ is a $\left(\delta, r_{0}\right)$-Reifenberg flat domain if it satisfies the following conditions:

(a) For every $x \in \partial \Omega$ and every $0<r \leq r_{0}$ there exists a hyperplane $\mathcal{P}(x, r)$ containing $x$ such that

$$
\operatorname{dist}_{H}(\partial \Omega \cap B(x, r), \mathcal{P}(x, r) \cap B(x, r)) \leq \delta r .
$$

(b) For every $x \in \partial \Omega$, one of the connected components of

$$
B\left(x, r_{0}\right) \cap\left\{x \in \mathbb{R}^{d+1}: \operatorname{dist}\left(x, \mathcal{P}\left(x, r_{0}\right)\right) \geq 2 \delta r_{0}\right\}
$$

is contained in $\Omega$ and the other is contained in $\mathbb{R}^{d+1} \backslash \Omega$.

If $\Omega$ is $\left(\delta, r_{0}\right)$-Reifenberg flat for every $r_{0}>0$, we say that it is $(\delta, \infty)$-Reifenberg flat.

Note that the topological condition (b) is asked only for the scale $r=r_{0}$. However, from the definition one can check that the same comparability condition also holds for $r \leq r_{0}$ (see [KT, Proposition 2.2] or [LMS, Lemma 5], for example). Further, we 
remark that the condition (b) is implied by (a) if one assumes that both $\Omega$ and $\partial \Omega$ are connected, as shown by David [Da].

We can now state the main result.

Theorem 1.2. For all $d \geq 2, \delta>0$ small enough and $r_{0}>0$, there is a $\left(\delta, r_{0}\right)$ Reifenberg flat domain $\Omega \subset \mathbb{R}^{d+1}$ and a set $E \subset \partial \Omega$ such that $\left.\mathcal{H}^{d}\right|_{\partial \Omega}$ is a Radon measure and if $\omega$ is the harmonic measure for $\Omega$ with respect to a fixed pole in $\Omega$, then

$$
\omega(E)>0=\mathcal{H}^{d}(E) .
$$

We give a sketch of the proof: We rely on the existence of Wolff snowflakes from either [W] or [LVV], which are NTA domains $\Omega \subset \mathbb{R}^{d+1}$ for which

$$
\lim _{r \rightarrow 0} \frac{\log \omega(B(\xi, r))}{\log r}<d \text { for } \omega \text { a.e. } \xi \in \partial \Omega .
$$

where $\omega$ denotes harmonic measure for $\Omega$ with respect to a fixed pole $z_{0} \in \Omega$. By some measure theory, this means we can find a compact set $E \subset \partial \Omega$ with $\omega(E)>0$ and constants $\alpha, r_{0}>0$ so that

$$
\omega(B(\xi, r))>r^{d-\alpha} \text { for all } r \in\left(0, r_{0}\right) .
$$

We then build a Reifenberg flat domain $\Omega^{+} \supset \Omega$ so that $\partial \Omega^{+} \supset E$ and use (1.2) to control the $\mathcal{H}^{d}$-measure of $\partial \Omega^{+}$. Moreover, if $\omega_{\Omega^{+}}$is harmonic measure for $\Omega^{+}$ with respect to the same pole $z_{0}$, then by the maximum principle, we have

$$
\omega_{\Omega^{+}}(E) \geq \omega(E)>0=\mathcal{H}^{d}(E) .
$$

The lemma for constructing this domain is not particular to our problem and may be of independent interest, see Section 2 .

As usual, in this paper we will use the letters $c, C$ to denote absolute constants which may change their values at different occurrences. Constants with subscripts, such as $c_{1}$, do not change their values at different occurrences. The notation $A \lesssim B$ means that there is some fixed constant $c$ such that $A \leq c B$. So $A \sim B$ is equivalent to $A \lesssim B \lesssim A$. If we want to write explicitly the dependence on some constants $c_{1}$ of the relationship such as "¿", we will write $A \lesssim c_{1} B$. We will assume all these implicit constants in these inequalities depend on $d$ and frequently omit the subscript.

We give many thanks to the anonymous referees for pointing out several errors and helping the authors improve the readability of this manuscript.

\section{The Enlarged DOMAin $\Omega_{\varepsilon}^{+}$}

We will assume $\Omega$ is $\left(\delta, r_{0}\right)$-Reifenberg flat for some $\delta<1 / 2$ sufficiently small. From now on, for $x \in \partial \Omega$ and $0<r \leq r_{0}$, we will denote by $N_{x, r}$ a unit vector, normal to $\mathcal{P}(x, r)$, with its sign chosen so that $x+\frac{3}{4} r N_{x, r} \notin \Omega$ and $x-\frac{3}{4} r N_{x, r} \in \Omega$. That $N_{x, r}$ can be taken in this way is guaranteed by the property (b) in the definition above, which holds for all $0<r \leq r_{0}$. In fact, from this one can deduce that

$$
B\left(x+\frac{3}{4} r N_{x, r}, \frac{r}{10}\right) \subset \Omega^{c} \quad \text { and } \quad B\left(x-\frac{3}{4} r N_{x, r}, \frac{r}{10}\right) \subset \Omega .
$$


Let us mention that, by Theorem 3.1 of [KT], there is $\delta_{0}=\delta_{0}(d)$ such that if $\Omega$ is $\left(\delta, r_{0}\right)$-Reifenberg flat, with $0<\delta \leq \delta_{0}$, then both $\Omega$ and $\mathbb{R}^{d+1} \backslash \bar{\Omega}$ are uniform domains.

Definition 2.1 (Whitney-type cubes). For an open set $\Omega \subsetneq \mathbb{R}^{d+1}$ that is $\left(\delta, r_{0}\right)$ Reifenberg flat and $K \geq 4$, we denote by $\mathcal{W}_{K}(\Omega)$ the set of maximal dyadic cubes $Q \subset \Omega$ such that $\operatorname{diam} K Q \leq r_{0}$ and $K Q \cap \Omega^{c}=\varnothing$. These cubes have disjoint interiors and can be easily shown to satisfy the following properties:

(a) $\min \left\{r_{0}, \operatorname{dist}\left(x, \Omega^{c}\right)\right\} / K \lesssim \ell(Q) \lesssim \min \left\{r_{0}, \operatorname{dist}\left(x, \Omega^{c}\right)\right\} / K$ for all $x \in Q$, where $\ell(Q)$ denotes the side length of the cube.

(b) If $Q, R \in \mathcal{W}_{K}(\Omega)$ and $\frac{K}{4} Q \cap \frac{K}{4} R \neq \varnothing$, then $\ell(Q) \sim_{K, d} \ell(R)$.

(c) $\sum_{Q \in \mathcal{W}_{k}(\Omega)} \chi_{\frac{K}{4} Q} \lesssim K, d \chi_{\Omega}$.

These are similar to the usual Whitney cubes, but we restrict their size.

Let $0<\varepsilon<1 / 100$ be some small constant and $E \subsetneq \partial \Omega$ be any closed set. Denote by $\mathcal{I}$ the family of cubes $Q \in \mathcal{W}_{\varepsilon^{-2}}\left(E^{c}\right)$ such that $Q \cap \partial \Omega \neq \varnothing$. Notice that

$$
\ell(Q) \lesssim \varepsilon^{2} \operatorname{dist}(Q, E) \quad \text { for all } Q \in \mathcal{I}
$$

and

$$
\partial \Omega \backslash E \subset \bigcup_{Q \in \mathcal{I}} Q
$$

For each $Q \in \mathcal{I}$, fix some point $z_{Q} \in Q \cap \partial \Omega$ and set

$$
B_{Q}=B\left(z_{Q}, \varepsilon \min \left\{r_{0}, \operatorname{dist}\left(z_{Q}, E\right)\right\}\right) .
$$

Notice that $\ell(Q) \sim \varepsilon r\left(B_{Q}\right)$. Then we consider the domain

$$
\Omega_{\varepsilon}^{+}=\Omega \cup \bigcup_{Q \in \mathcal{I}} B_{Q} .
$$

Our main objective in this section consists in proving the following.

Lemma 2.2. Let $r_{0} \in(0, \infty]$ and let $\varepsilon>0$ be small enough. There exists $\delta_{0}=$ $\delta_{0}(\varepsilon)>0$ such that if $\Omega \subset \mathbb{R}^{d+1}$ is $\left(\delta, r_{0}\right)$-Reifenberg flat for some $\delta \in\left(0, \delta_{0}\right), E \subsetneq \partial \Omega$ is closed and $\Omega_{\varepsilon}^{+}$is as above, then $E \subset \partial \Omega_{\varepsilon}^{+}$and $\Omega_{\varepsilon}^{+}$is $\left(c \varepsilon^{1 / 2}, r_{0} / 2\right)$-Reifenberg flat.

First we will prove the following auxiliary result.

Lemma 2.3. With the same notation and assumptions as in Lemma 2.2, for every $Q \in \mathcal{I}$, there exists a function $f_{Q}: \mathcal{P}\left(z_{Q}, 30 r\left(B_{Q}\right)\right) \cap 10 B_{Q} \rightarrow \mathcal{P}\left(z_{Q}, 30 r\left(B_{Q}\right)\right)^{\perp}$ such that, assuming after a suitable rotation that $L_{Q}:=\mathcal{P}\left(z_{Q}, 30 r\left(B_{Q}\right)\right)=\mathbb{R}^{d} \times\{0\}$, the following holds:

(a) $\Omega_{\varepsilon}^{+} \cap 10 B_{Q}=\left\{x \in 10 B_{Q}: x_{d+1}<f_{Q}(\tilde{x})\right\}$, where $\widetilde{x}=\left(x_{1}, \ldots, x_{d}\right)$.

(b) For all $\widetilde{x} \in \mathcal{P}\left(z_{Q}, 30 r\left(B_{Q}\right)\right) \cap 10 B_{Q},\left|f_{Q}(\widetilde{x})-r\left(B_{Q}\right)\right| \leq c \varepsilon r\left(B_{Q}\right)$.

(c) The function $f_{Q}$ is Lipschitz with Lipschitz constant at most $c \varepsilon^{1 / 2}$.

(d) For all $x \in 10 B_{Q} \cap L_{Q},\left(x, f_{Q}(x)\right) \in \partial \Omega_{\varepsilon}^{+}$ 
Proof. We will assume that $\delta \ll \varepsilon$. To simplify notation we write

$$
r_{Q}=r\left(B_{Q}\right)=\varepsilon \min \left\{r_{0}, \operatorname{dist}\left(z_{Q}, E\right)\right\} .
$$

Further, we suppose that the component $\left\{x \in 30 B_{Q}: x_{d+1}<-60 \delta r_{Q}\right\}$ is contained in $\Omega$ (recall that the property (b) in Definition 1.1 holds for all $r \leq r_{0}$ ).

Claim 1. There is a finite subfamily $\mathcal{I}_{Q} \subset \mathcal{I}$ such that

$$
20 B_{Q} \cap \partial \Omega_{\varepsilon}^{+} \subset \bigcup_{P \in \mathcal{I}_{Q}} \partial B_{P}
$$

Further, for every $P \in \mathcal{I}_{Q}$, we have

$$
\begin{gathered}
\left|z_{P}-z_{Q}\right| \leq 30 r_{Q}, \\
\operatorname{dist}\left(z_{P}, L_{Q}\right) \leq 30 \delta r_{Q},
\end{gathered}
$$

and

$$
\left|r_{P}-r_{Q}\right| \leq c_{1} \varepsilon r_{Q}
$$

Indeed, $\partial \Omega_{\varepsilon}^{+} \subset E \cup \bigcup_{P \in \mathcal{I}} \partial B_{P}$ holds by definition, which obviously implies

$$
\partial \Omega_{\varepsilon}^{+} \backslash E \subset \bigcup_{P \in \mathcal{I}} \partial B_{P}
$$

We denote by $\mathcal{I}_{Q}$ the subfamily of the cubes $P \in \mathcal{I}$ such that $20 B_{Q} \cap B_{P} \neq \varnothing$, so that

$$
20 B_{Q} \cap \partial \Omega_{\varepsilon}^{+} \subset E \cup \bigcup_{P \in \mathcal{I}_{Q}} \partial B_{P}
$$

By definition, $E \cap 20 B_{Q}=\varnothing$, from which (2.2) readily follows from (2.6) and (2.7).

Suppose that $P \in \mathcal{I}_{Q}$. Also, by the definition of $r_{P}$ and $r_{Q}$ along with $20 B_{Q} \cap B_{P}$, and since $\min \left\{r_{0}, \operatorname{dist}(z, E)\right\}$ is 1-Lipschitz, we have

$$
\left|r_{P}-r_{Q}\right| \leq \varepsilon\left|z_{P}-z_{Q}\right| \leq \varepsilon\left(r_{P}+20 r_{Q}\right) \text {. }
$$

One can check that this ensures that

$$
\left|r_{P}-r_{Q}\right| \leq c \varepsilon r_{Q},
$$

and thus (2.5) holds. This implies that $r_{P} \sim r_{Q}$, and thus $\ell(P) \sim \varepsilon r_{Q}$. From this condition, taking into account that the cubes $P \in \mathcal{I}_{Q}$ are pairwise disjoint and all of them intersect $20 B_{Q}$ (by the definition of $\mathcal{I}_{Q}$ ), it follows that $\mathcal{I}_{Q}$ is finite.

Since for $\varepsilon$ small enough we have $r_{P} \leq 2 r_{Q}$, we deduce that

$$
\left|z_{P}-z_{Q}\right| \leq r_{P}+20 r_{Q} \leq 22 r_{Q}
$$

which yields (2.3). On the other hand, (2.4) follows from the Reifenberg flatness of $\Omega$ and the fact that $z_{P} \in \partial \Omega \cap 30 B_{Q}$. 
Claim 2. If $x \in 20 B_{Q} \cap \partial \Omega_{\varepsilon}^{+}$, then

$$
\left|x_{d+1}-r_{Q}\right| \leq c_{2} \varepsilon r_{Q}
$$

for some absolute constant $c_{2}>0$. Moreover, every $x \in 20 B_{Q} \cap \partial \Omega_{\varepsilon}^{+}$satisfies

$$
\left(1-c_{2} \varepsilon\right) r_{Q} \leq x_{d+1} \leq\left(1+c_{2} \varepsilon\right) r_{Q}
$$

Let $x \in 20 B_{Q} \cap \partial \Omega_{\varepsilon}^{+}$. Notice first that, by (2.2), (2.4) and (2.5),

$$
\left|x_{d+1}\right|=\operatorname{dist}\left(x, L_{Q}\right) \leq \max _{P \in \mathcal{I}_{Q}}\left[\operatorname{dist}\left(z_{P}, L_{Q}\right)+r_{P}\right] \leq 30 \delta r_{Q}+\left(1+c_{1} \varepsilon\right) r_{Q} \leq\left(1+2 c_{1} \varepsilon\right) r_{Q},
$$

since we are assuming $\delta \ll \varepsilon$.

Now we will show that

$$
\left|x_{d+1}\right|=\operatorname{dist}\left(x, L_{Q}\right) \geq\left(1-c_{2} \varepsilon\right) r_{Q} .
$$

To this end, notice that, by the Reifenberg flatness of $\Omega$, if $\widetilde{x}$ is the projection of $x$ onto $L_{Q}$, there exists $x^{\prime} \in \partial \Omega$ such that $\left|x^{\prime}-\widetilde{x}\right| \leq 30 \delta r_{Q}$. Let $P \in \mathcal{I}_{Q}$ be the Whitney cube such that $x^{\prime} \in P$. Then,

$$
\left|x-z_{P}\right| \leq|x-\widetilde{x}|+\left|\widetilde{x}-x^{\prime}\right|+\left|x^{\prime}-z_{P}\right| \leq\left|x_{d+1}\right|+30 \delta r_{Q}+c \ell(P) .
$$

Recalling that $\ell(P) \sim \ell(Q) \sim \varepsilon r_{Q}$ and using that $\left|x-z_{P}\right| \geq r_{P}$ (because $x$ is not in the interior of $\left.B_{P}\right)$, we deduce that

$\left|x_{d+1}\right| \geq\left|x-z_{P}\right|-30 \delta r_{Q}-c \ell(P) \geq r_{P}-30 \delta r_{Q}-c \varepsilon r_{Q} \geq\left(1-c_{1} \varepsilon-30 \delta-c \varepsilon\right) r_{Q}$, which proves (2.12) since we assume that $\delta \ll \varepsilon$.

From (2.12), we infer that

$$
x \in 30 B_{Q} \backslash U_{60 \delta r_{Q}}\left(L_{Q}\right),
$$

using again that $\delta \ll \varepsilon \ll 1$, where $U_{\epsilon}(F)$ stands for the $\epsilon$-neighborhood of the set $F$. Since $x \in \Omega^{c}$ (as $\partial \Omega_{\varepsilon}^{+} \subset \Omega^{c}$ ), we infer that $x_{d+1}>0$ (because we are assuming that $\left\{x \in 30 B_{Q}: x_{d+1}<-60 \delta r_{Q}\right\}$ is contained in $\Omega$ by Definition 1.1 and (2.1)). Hence $\left|x_{d+1}\right|=x_{d+1}$ and then (2.11) and (2.12) yield (2.9).

The second statement in the claim follows easily from (2.9).

Claim 3. For $\widetilde{x} \in 10 B_{Q} \cap L_{Q}$, let

$$
f_{Q}(\widetilde{x})=\max \left\{t \in \mathbb{R}:(\widetilde{x}, t) \in 20 \overline{B_{Q}} \cap \overline{\Omega_{\varepsilon}^{+}}\right\} .
$$

The function $f_{Q}: 10 B_{Q} \cap L_{Q} \rightarrow \mathbb{R}$ is well defined, $\left(\widetilde{x}, f_{Q}(\widetilde{x})\right) \in \partial \Omega_{\varepsilon}^{+}$, and

$$
\left|f_{Q}(\widetilde{x})-r_{Q}\right| \leq c_{2} \varepsilon r_{Q}
$$

To see this, let $\widetilde{x} \in 10 B_{Q} \cap L_{Q}$ and consider the points $x_{1}=\left(\widetilde{x},-2 r_{Q}\right)$ and $x_{2}=\left(\widetilde{x}, 2 r_{Q}\right)$. Then we have

$$
x_{1} \in 20 B_{Q} \cap \Omega \subset 20 B_{Q} \cap \Omega_{\varepsilon}^{+} \quad \text { and } \quad x_{2} \in 20 B_{Q} \backslash \Omega_{\varepsilon}^{+} .
$$

The first statement follows from the fact that $\left\{x \in 30 B_{Q}: x_{d+1}<-60 \delta r_{Q}\right\}$ is contained in $\Omega$ and the second one from (2.10). Hence, there exists some $t_{0} \in$ $\left[-2 r_{Q}, 2 r_{Q}\right]$ such that $\left(\widetilde{x}, t_{0}\right) \in \partial \Omega_{\varepsilon}^{+}$, and thus the maximum in (2.13) is taken over 
a non-empty set. Further, (2.10) also tells us that $(\widetilde{x}, t) \notin \Omega_{\varepsilon}^{+}$for every $t \geq 2 r_{Q}$, and thus it follows that $\left(\widetilde{x}, f_{Q}(\widetilde{x})\right) \in \partial \Omega_{\varepsilon}^{+}$.

The estimate (2.14) is an immediate consequence of (2.9).

Claim 4. Let $x=(\widetilde{x}, t) \in 10 B_{Q}$ with $t<f_{Q}(\widetilde{x})$. Then $x \in \Omega_{\varepsilon}^{+}$.

By Claim 1 and Claim 3, there exists some $P \in \mathcal{I}_{Q}$ such that $\left(\widetilde{x}, f_{Q}(\widetilde{x})\right) \in \partial B_{P}$. Let $\widetilde{L}_{Q}$ be a hyperplane parallel to $L_{Q}$ passing through $z_{P}$. Let $y \in \mathbb{R}^{d+1}$ be the reflection of $\left(\widetilde{x}, f_{Q}(\widetilde{x})\right)$ with respect to $\widetilde{L}_{Q}$. It is clear that $y \in \partial B_{P}$ and thus the (open) segment with end points $\left(\widetilde{x}, f_{Q}(\widetilde{x})\right)$ and $y$ is contained in $B_{P}$ and thus in $\Omega_{\varepsilon}^{+}$. That is to say,

$$
(\widetilde{x}, t) \in \Omega_{\varepsilon}^{+} \quad \text { if } t \in\left(y_{d+1}, f_{Q}(\widetilde{x})\right) .
$$

By symmetry, $y_{d+1}+f_{Q}(\widetilde{x}) \leq 2 \operatorname{dist}\left(z_{P}, L_{Q}\right)$ and $\operatorname{since} \operatorname{dist}\left(z_{P}, L_{Q}\right) \leq 30 \delta r_{Q} \leq \varepsilon r_{Q}$, using also (2.14) we infer that

$$
y_{d+1} \leq-f_{Q}(\widetilde{x})+2 \varepsilon r_{Q} \leq-(1-c \varepsilon) r_{Q} \leq-\frac{1}{2} r_{Q}
$$

On the other, since $(\widetilde{x}, t) \in \Omega \subset \Omega_{\varepsilon}^{+}$for $(\widetilde{x}, t) \in 10 B_{Q}$ with $t<-r_{Q} / 2$, the claim follows.

Claim 5. The function $f_{Q}$ is Lipschitz with Lipschitz constant not exceeding $c \varepsilon^{1 / 2}$.

From Claim 3 and Claim 1 we deduce that

$$
f_{Q}(\widetilde{x})=\max \left\{t: \exists P \in \mathcal{I}_{Q} \text { such that }(\widetilde{x}, t) \in \partial B_{P}\right\} .
$$

Observe that if $x=f(\tilde{x}) \in \partial B_{P} \cap \partial \Omega_{\varepsilon}^{+}$, then

$$
f_{Q}(\widetilde{x})=\sqrt{r_{P}^{2}-\left|\widetilde{x}-\widetilde{z}_{P}\right|^{2}}+z_{P, d}
$$

where we wrote $z_{P}=\left(\widetilde{z}_{P}, z_{P, d}\right)$. Further, since $f_{Q}(\widetilde{x}) \geq\left(1-c_{2} \varepsilon\right) r_{Q}$ by $(2.14)$, clearly we can write $f_{Q}(\tilde{x})=g_{P}(\tilde{x})$, where $g_{P}: L_{Q} \rightarrow L_{Q}^{\perp}$ is defined by

$$
g_{P}(\widetilde{x})=\max \left(\sqrt{r_{P}^{2}-\left|\widetilde{x}-\widetilde{z}_{P}\right|^{2}}+z_{P, d},\left(1-c_{2} \varepsilon\right) r_{Q}\right) \quad \text { if }\left|\widetilde{x}-\widetilde{z}_{P}\right| \leq r_{P},
$$

and $g_{P}(\widetilde{x})=\left(1-c_{2} \varepsilon\right) r_{Q}$ otherwise. From (2.15) we infer that, for all $\widetilde{x} \in L_{Q} \cap 10 B_{Q}$,

$$
f_{Q}(\widetilde{x})=\max _{P \in \mathcal{I}_{Q}} g_{P}(\widetilde{x}) .
$$

So to prove the claim it suffices to show that each function $g_{P}$ is Lipschitz with Lipschitz constant at most $c \varepsilon^{1 / 2}$. To check that this holds, notice that $g_{P}$ is continuous and differentiable a.e., with

$$
\left|\nabla g_{P}(\widetilde{x})\right|=\frac{\left|\widetilde{x}-\widetilde{z}_{P}\right|}{\sqrt{r_{P}^{2}-\left|\widetilde{x}-\widetilde{z}_{P}\right|^{2}}} \quad \text { if } \sqrt{r_{P}^{2}-\left|\widetilde{x}-\widetilde{z}_{P}\right|^{2}}+z_{P, d}>\left(1-c_{2} \varepsilon\right) r_{Q}
$$

and $\left|\nabla g_{P}(\widetilde{x})\right|=0$ a.e. otherwise. The condition on the right hand side of (2.16) implies that

$$
\sqrt{r_{P}^{2}-\left|\widetilde{x}-\widetilde{z}_{P}\right|^{2}}>\left(1-c_{2} \varepsilon\right) r_{Q}-z_{P, d} \geq\left(1-c_{3} \varepsilon\right) r_{P}
$$


by (2.5) and (2.4). This gives

$$
\left|\widetilde{x}-\widetilde{z}_{P}\right|^{2}<r_{P}^{2}-\left(1-c_{3} \varepsilon\right)^{2} r_{P}^{2} \leq 2 c_{3} \varepsilon r_{P}^{2} .
$$

Plugging this estimate into (2.16) we get

$$
\left|\nabla g_{P}(\widetilde{x})\right| \leq c \varepsilon^{1 / 2}
$$

which implies that $\operatorname{Lip}\left(g_{P}\right) \leq c \varepsilon^{1 / 2}$, as wished. This concludes the proof of the claim.

The lemma follows from the statements in the claims above.

Proof of Lemma 2.2. First we show that $E \subset \partial \Omega_{\varepsilon}^{+}$. Notice that $E \subset \bar{\Omega} \subset \overline{\Omega_{\varepsilon}^{+}}$. So it suffices to show that for each $x \in E$ there exists a sequence of points $\left\{x_{k}\right\}_{k} \subset\left(\Omega_{\varepsilon}^{+}\right)^{c}$ such that $x_{k} \rightarrow x$ as $k \rightarrow \infty$. To construct this sequence, for each $0<r \leq r_{0}$ consider the ball $B(x, r)$, so that by the Reifenberg flatness of $\Omega, \partial \Omega \cap B(x, r) \subset U_{\delta r}(\mathcal{P}(x, r))$. Further, any ball $B_{Q}$, with $Q \in \mathcal{I}$, which intersects $B(x, r)$ satisfies

$$
r_{Q} \leq \varepsilon \operatorname{dist}\left(z_{Q}, E\right) \leq \varepsilon\left|z_{Q}-x\right| \leq \varepsilon\left(\sqrt{d+1} r_{Q}+r\right),
$$

where as in the previous lemma, we write $r_{Q}=r\left(B_{Q}\right) \leq \varepsilon \operatorname{dist}\left(z_{Q}, E\right)$. Then it follows that $r_{Q}<2 \varepsilon r$ for $\varepsilon$ small. Hence we deduce that $B(x, r) \cap \overline{\Omega_{\varepsilon}^{+}} \subset B(x, r) \cap$ $U_{2 \varepsilon r}(\bar{\Omega})$. From (2.1), we deduce that if $\varepsilon$ is small enough, then

$$
y_{r}:=x+\frac{3}{4} r N_{x, r} \in\left(\Omega_{\varepsilon}^{+}\right)^{c} .
$$

Thus, setting $r=1 / k$ and $x_{k}=y_{1 / k}$, we are done.

Now we have to show that $\Omega_{\varepsilon}^{+}$is $\left(c \varepsilon^{1 / 2}, r_{0} / 2\right)$-Reifenberg flat. By construction, if $x \in \partial \Omega_{\varepsilon}^{+}$, then either $x \in E$ or there exists some ball $B_{Q}$ such that $x \in \partial B_{Q}$, and so $\operatorname{dist}(x, \partial \Omega) \leq r_{Q}$.

To show that the properties (a) and (b) in the Definition 1.1 hold for $\Omega_{\varepsilon}^{+}$and the ball $B(x, r)$, with $0<r<r_{0} / 2$ and with $c \varepsilon^{1 / 2}$ instead of $\delta$, we distinguish several cases:

Case 1. Suppose that $r \geq \varepsilon^{-1 / 2} r_{Q}$ for every $Q \in \mathcal{I}$ such that

$$
B_{Q} \cap B(x, 2 r) \neq \varnothing .
$$

From the discussion in the previous paragraphs, it turns out that there exists $x^{\prime} \in \partial \Omega$ such that

$$
\left|x-x^{\prime}\right| \leq \sup \left\{r_{Q}: Q \in \mathcal{I}, B_{Q} \cap B(x, r) \neq \varnothing\right\} \leq \varepsilon^{1 / 2} r .
$$


Let $L$ be the hyperplane parallel to $\mathcal{P}\left(x^{\prime}, r\right)$ that contains $x$. Then we set

$$
\begin{aligned}
\operatorname{dist}_{H}\left(\partial \Omega_{\varepsilon}^{+} \cap B(x, r), L \cap B(x, r)\right) \leq & \operatorname{dist}_{H}\left(\partial \Omega_{\varepsilon}^{+} \cap B(x, r), \partial \Omega \cap B(x, r)\right) \\
& +\operatorname{dist}_{H}\left(\partial \Omega \cap B(x, r), \mathcal{P}\left(x^{\prime}, r\right) \cap B(x, r)\right) \\
& +\operatorname{dist}_{H}\left(\mathcal{P}\left(x^{\prime}, r\right) \cap B(x, r), L \cap B(x, r)\right) \\
= & 1+2+3 .
\end{aligned}
$$

It is immediate to check that

$$
\text { (3) } \leq c\left|x-x^{\prime}\right| \leq c \varepsilon^{1 / 2} r \text {. }
$$

On the other hand, to estimate 2 we write

$$
\begin{aligned}
2 \leq & \operatorname{dist}_{H}\left(\partial \Omega \cap B(x, r), \partial \Omega \cap B\left(x^{\prime}, r\right)\right)+\operatorname{dist}_{H}\left(\partial \Omega \cap B\left(x^{\prime}, r\right), \mathcal{P}\left(x^{\prime}, r\right) \cap B\left(x^{\prime}, r\right)\right) \\
& +\operatorname{dist}_{H}\left(\mathcal{P}\left(x^{\prime}, r\right) \cap B\left(x^{\prime}, r\right), \mathcal{P}\left(x^{\prime}, r\right) \cap B(x, r)\right) .
\end{aligned}
$$

It is easy to check that the first and the last terms on the right hand side above do not exceed $c\left|x-x^{\prime}\right| \leq c \varepsilon^{1 / 2} r$, while

$$
\operatorname{dist}_{H}\left(\partial \Omega \cap B\left(x^{\prime}, r\right), \mathcal{P}\left(x^{\prime}, r\right) \cap B\left(x^{\prime}, r\right)\right) \leq \delta r,
$$

by the Reifenberg flatness of $\Omega$. Thus,

$$
2 \lesssim\left(\varepsilon^{1 / 2}+\delta\right) r \lesssim \varepsilon^{1 / 2} r
$$

assuming $\delta \leq \varepsilon^{1 / 2}$

To estimate (1) we use the fact that for every $y \in \partial \Omega_{\varepsilon}^{+} \cap B(x, r)$ there exists $y^{\prime} \in \partial \Omega$ such that $\left|y-y^{\prime}\right| \leq \varepsilon^{1 / 2} r$, and also for every $z^{\prime} \in \partial \Omega \cap B(x, r)$ there exists some $z \in \partial \Omega_{\varepsilon}^{+}$such that $\left|z-z^{\prime}\right| \leq c \varepsilon^{1 / 2} r$. The existence of $y^{\prime}$ follows by arguing as in (2.18), while the existence of $z$ can be shown with the aid of Lemma 2.3. From these facts one can derive that

$$
\text { (1) } \lesssim \varepsilon^{1 / 2} r \text {. }
$$

If we gather the estimates for (1), 2) and (3), we get

$$
\operatorname{dist}_{H}\left(\partial \Omega_{\varepsilon}^{+} \cap B(x, r), L \cap B(x, r)\right) \lesssim \varepsilon^{1 / 2} r .
$$

We claim now that one of the connected components of

$$
B(x, r) \cap\left\{y \in \mathbb{R}^{d+1}: \operatorname{dist}(y, L) \geq c \varepsilon^{1 / 2} r\right\}
$$

is contained in $\Omega_{\varepsilon}^{+}$and the other is contained in $\mathbb{R}^{d+1} \backslash \Omega_{\varepsilon}^{+}$. To see this, pick $x^{\prime} \in \partial \Omega$ closest to $x$. We take into account that, for $\delta$ small enough, and since $\operatorname{dist}\left(L, P\left(x^{\prime}, r\right)\right)=\left|x-x^{\prime}\right| \leq \varepsilon^{1 / 2} r$,

$$
U_{2 \delta r}\left(\mathcal{P}\left(x^{\prime}, r\right)\right) \subset U_{c \varepsilon^{1 / 2} r}(L),
$$

and thus by the property (b) in the Definition 1.1 applied to $\Omega, x^{\prime}$ and $2 r$, one of the components of $B\left(x, \frac{3}{2} r\right) \backslash U_{c \varepsilon^{1 / 2} r}(L)$ is contained in $\Omega$ and the other in $\mathbb{R}^{d+1} \backslash \Omega$. 
Since all the balls $B_{Q}$ which intersect $B(x, r)$ have radius at most $\varepsilon^{1 / 2} r$ and they intersect $\partial \Omega$, we infer that all of them are contained in $U_{c^{\prime} \varepsilon^{1 / 2} r}(L)$, and then

$$
\left(B(x, r) \backslash U_{c^{\prime} \varepsilon^{1 / 2} r}(L)\right) \cap \Omega=\left(B(x, r) \backslash U_{c^{\prime} \varepsilon^{1 / 2} r}(L)\right) \cap \Omega_{\varepsilon}^{+}
$$

and

$$
\left(B(x, r) \backslash U_{c^{\prime} \varepsilon^{1 / 2} r}(L)\right) \cap \Omega^{c}=\left(B(x, r) \backslash U_{c^{\prime} \varepsilon^{1 / 2} r}(L)\right) \cap\left(\Omega_{\varepsilon}^{+}\right)^{c},
$$

which implies that (b) in the Definition 1.1 holds for $\Omega_{\varepsilon}^{+}, x$ and $r$.

Case 2. Suppose that $r_{Q}<r<\varepsilon^{-1 / 2} r_{Q}$ for some $Q \in \mathcal{I}$ such that $B_{Q} \cap B(x, 2 r) \neq$ $\varnothing$. For this case, we will require the following lemma that will shorten some computations.

Lemma 2.4. For a closed set $E, x \in E, r>0$, and a d-plane $P$ intersecting $B(x, r)$, we have

$\max \left\{\sup _{y \in E \cap B(x, r)} \operatorname{dist}(y, P), \sup _{y \in P \cap B(x, r)} \operatorname{dist}(y, E)\right\} \sim \operatorname{dist}_{H}(E \cap B(x, r), P \cap B(x, r))$.

We leave the details to the reader.

We denote

$$
\widetilde{L}_{Q}=L_{Q}+r_{Q} N_{z_{Q}, r_{Q}}
$$

where again $L_{Q}=\mathcal{P}\left(z_{Q}, 30 r_{Q}\right)$. We will show that, for the point $x \in \partial \Omega_{\varepsilon}^{+}$,

$$
\operatorname{dist}_{H}\left(\partial \Omega_{\varepsilon}^{+} \cap B(x, r), \widetilde{L}_{Q} \cap B(x, r)\right) \lesssim \varepsilon^{1 / 2} r .
$$

Although we cannot guarantee that $x \in \widetilde{L}_{Q}$, it is clear that from this estimate one deduces that (a) from Definition 1.1 holds just by translating $\widetilde{L}_{Q}$ appropriately.

To prove (2.19) first we claim that if $B_{P} \cap B(x, 3 r) \neq \varnothing$ for some $P \in \mathcal{I}$, then $\ell(P) \sim \ell(Q)$ and

$$
\left|r_{P}-r_{Q}\right| \lesssim \varepsilon^{1 / 2} r_{Q}
$$

To see this, notice that

$$
\operatorname{dist}\left(B_{P}, B_{Q}\right) \leq 5 r \leq 5 \varepsilon^{-1 / 2} r_{Q} .
$$

Then, recalling that $r_{P} \leq \varepsilon \operatorname{dist}\left(z_{P}, E\right)$ for all $P \in \mathcal{I}$, we get

$$
\left|r_{Q}-r_{P}\right| \leq \varepsilon\left|z_{P}-z_{Q}\right| \leq \varepsilon\left(r_{P}+r_{Q}+5 \varepsilon^{-1 / 2} r_{Q}\right) \lesssim \varepsilon^{1 / 2}\left(r_{Q}+r_{P}\right) .
$$

In particular, this implies that $r_{Q} \sim r_{P}$ and so that $\ell(P) \sim \ell(Q)$ and finishes the proof of the claim.

We will need the following well known lemma, a proof of which is supplied in [AT].

Lemma 2.5. Suppose $P_{1}$ and $P_{2}$ are $n$-planes in $\mathbb{R}^{d+1}$ and $X=\left\{x_{0}, \ldots, x_{n}\right\}$ are points so that

(a) $\eta=\eta(X)=\min _{i} \operatorname{dist}\left(x_{i}, \operatorname{span}\left(X \backslash\left\{x_{i}\right\}\right)\right) / \operatorname{diam} X \in(0,1)$ and

(b) $\operatorname{dist}\left(x_{i}, P_{j}\right)<\theta \operatorname{diam} X$ for $i=0, \ldots, n$ and $j=1,2$, where $\theta<\eta(d+1)^{-1} / 2$. 
Then

$$
\operatorname{dist}\left(y, P_{1}\right) \leq \theta\left(\frac{2 d}{\eta} \operatorname{dist}(y, X)+\operatorname{diam} X\right) \text { for all } y \in P_{2} \text {. }
$$

We continue now with the proof of (2.19). To this end, denote $L=\mathcal{P}\left(z_{Q}, 30 r\right)$ and assume

$$
B_{P} \cap B(x, 3 r) \neq \emptyset \text {. }
$$

Let $x_{0}=z_{P}$ and $x_{1}, \ldots, x_{d} \in L_{P}$ be such that $\left|x_{i}-x_{0}\right|=r_{P}$ for all $i=1, \ldots, d$ and $X=\left\{x_{0}, . ., x_{d}\right\}$ is a scaled copy of the vectors $\left\{e_{0}, \ldots, e_{d}\right\} \subset \mathbb{R}^{d}$ where $e_{0}=0$ and $e_{1}, \ldots, e_{d}$ are the standard basis vectors. Then it is not hard to show that $\eta\left(\left\{x_{0}, \ldots, x_{d}\right\}\right)=\eta\left(\left\{e_{0}, \ldots, e_{d}\right\}\right) \sim_{d} 1$ and diam $X \sim_{d} r_{P}$. By the definition of $L_{P}$, there are $x_{i}^{\prime} \in \partial \Omega$ with $\left|x_{i}-x_{i}^{\prime}\right|<30 \delta r_{P} \leq 30 \delta r$, and so for each $i=0, \ldots, d$, by (2.17) and (2.23),

$x_{i}^{\prime} \in B\left(z_{P}, r_{P}+30 \delta r_{P}\right) \subseteq B(x, 3 r+30 \delta r) \subseteq B\left(z_{Q}, r_{Q}+2 r+3 r+30 \delta r\right) \subseteq B\left(z_{Q}, 7 r\right)$

Thus, by the definition of $L$, there are $x_{i}^{\prime \prime} \in L$ so that $\left|x_{i}^{\prime}-x_{i}^{\prime \prime}\right|<30 \delta r$, hence

$$
\operatorname{dist}\left(x_{i}, L\right) \leq\left|x_{i}-x_{i}^{\prime \prime}\right|<60 \delta r \lesssim \varepsilon^{-1 / 2} \delta r_{P} \sim \delta \varepsilon^{-1 / 2} \operatorname{diam} X
$$

and thus the previous lemma implies, for $B_{P} \cap B(x, 3 r) \neq \varnothing$,

$$
\operatorname{dist}_{H}\left(L \cap B(x, 30 r), L_{P} \cap B(x, 30 r)\right) \leq c \delta \varepsilon^{-1 / 2} r<\varepsilon r .
$$

Then, assuming $\delta \ll \varepsilon$, we deduce that

$$
\operatorname{dist}_{H}\left(L \cap 10 B_{P}, L_{P} \cap 10 B_{P}\right) \leq \operatorname{dist}_{H}\left(L \cap B(x, 30 r), L_{P} \cap B(x, 30 r)\right) \leq \varepsilon r .
$$

In particular, this also holds for $P=Q$, and thus we have

$$
\begin{aligned}
\operatorname{dist}_{H}\left(L_{Q} \cap 10 B_{P}, L_{P} \cap 10 B_{P}\right) \leq & \operatorname{dist}_{H}\left(L_{Q} \cap 10 B_{P}, L \cap 10 B_{P}\right) \\
& +\operatorname{dist}_{H}\left(L \cap 10 B_{P}, L_{P} \cap 10 B_{P}\right) \\
\leq & 2 \varepsilon r \lesssim \varepsilon^{1 / 2} r_{P} .
\end{aligned}
$$

Given $P$ as above, we consider the hyperplanes

$$
\widetilde{L}_{P}=L_{P}+r_{P} N_{z_{P}, r_{P}}, \quad \widetilde{L}_{Q, P}=L_{Q}+r_{P} N_{z_{P}, r_{P}} .
$$

Notice that, by Lemmas 2.3 and 2.4,

$$
\operatorname{dist}_{H}\left(\widetilde{L}_{P} \cap 10 B_{P}, \partial \Omega_{\varepsilon}^{+} \cap 10 B_{P}\right) \lesssim \varepsilon r_{P}
$$

We write

$$
\begin{aligned}
\operatorname{dist}_{H}\left(\widetilde{L}_{Q} \cap 10 B_{P}, \widetilde{L}_{P} \cap 10 B_{P}\right) \leq & \operatorname{dist}_{H}\left(\widetilde{L}_{Q} \cap 10 B_{P}, \widetilde{L}_{Q, P} \cap 10 B_{P}\right) \\
& +\operatorname{dist}_{H}\left(\widetilde{L}_{Q, P} \cap 10 B_{P}, \widetilde{L}_{P} \cap 10 B_{P}\right) .
\end{aligned}
$$

From (2.25), it follows easily that $\left|N_{z_{P}, r_{P}}-N_{z_{Q}, r_{Q}}\right| \lesssim \varepsilon^{1 / 2}$. Taking also into account that $\left|r_{P}-r_{Q}\right| \lesssim \varepsilon^{1 / 2} r_{Q} \leq \varepsilon^{1 / 2} r$ by $(2.20)$, it is easy to check that

$\operatorname{dist}_{H}\left(\widetilde{L}_{Q} \cap 10 B_{P}, \widetilde{L}_{Q, P} \cap 10 B_{P}\right) \lesssim\left|r_{P} N_{z_{P}, r_{P}}-r_{Q} N_{z_{Q}, r_{Q}}\right| \lesssim \varepsilon^{1 / 2} r_{Q}+\varepsilon^{1 / 2} r \lesssim \varepsilon^{1 / 2} r$ 
and, by (2.25),

$$
\operatorname{dist}_{H}\left(\widetilde{L}_{Q, P} \cap 10 B_{P}, \widetilde{L}_{P} \cap 10 B_{P}\right) \lesssim \operatorname{dist}_{H}\left(L_{Q} \cap 10 B_{P}, L_{P} \cap 10 B_{P}\right) \lesssim \varepsilon r .
$$

So we deduce from the above two inequalities that

$$
\operatorname{dist}_{H}\left(\widetilde{L}_{Q} \cap 10 B_{P}, \widetilde{L}_{P} \cap 10 B_{P}\right) \lesssim \varepsilon^{1 / 2} r .
$$

Together with (2.26), this gives

$$
\operatorname{dist}_{H}\left(\partial \Omega_{\varepsilon}^{+} \cap 10 B_{P}, \widetilde{L}_{Q} \cap 10 B_{P}\right) \lesssim \varepsilon^{1 / 2} r+\varepsilon r_{P} \lesssim \varepsilon^{1 / 2} r .
$$

To see that (2.19) holds, by Lemma 2.4 it suffices to show that

$$
\text { for all } y \in \partial \Omega_{\varepsilon}^{+} \cap B(x, r) \text { there exists } y^{\prime} \in \widetilde{L}_{Q} \text { such that }\left|y-y^{\prime}\right| \lesssim \varepsilon^{1 / 2} r \text {, }
$$

and

$$
\text { for all } y \in \widetilde{L}_{Q} \cap B(x, r) \text { there exists } y^{\prime} \in \partial \Omega_{\varepsilon}^{+} \text {such that }\left|y-y^{\prime}\right| \lesssim \varepsilon^{1 / 2} r .
$$

The statement (2.28) holds because of (2.27) and the fact that

$$
\partial \Omega_{\varepsilon}^{+} \cap B(x, r) \subset \bigcup_{P \in \mathcal{I}: P \cap B(x, r) \neq \varnothing} B_{P}
$$

Indeed, it is easy to see that, under our current assumptions, $x \in E^{c}$ and by virtue of (2.20) we have that $\partial \Omega_{\varepsilon}^{+} \cap B(x, r) \subset E^{c}$. To prove (2.29), given $y \in \widetilde{L}_{Q} \cap B(x, r)$, let $z$ be the orthogonal projection of $y$ on $L_{Q}$, so that $|y-z|=r_{Q}$, hence $z \in B\left(x, r+r_{Q}\right)$. By (2.24) (with $P=Q$ and $\varepsilon$ small enough), we may find $\widetilde{z} \in B(x, 30 r) \cap L$ and $|\widetilde{z}-z|<\varepsilon r$, and by the definition of $L$ and for $\delta$ small, we can find $z^{\prime} \in \partial \Omega$ with $\left|z^{\prime}-\tilde{z}\right|<30 \delta r<\varepsilon r$, and so $\left|z-z^{\prime}\right|<2 \varepsilon r$. Let $P \in \mathcal{I}$ be such that $z^{\prime} \in B_{P}$. Notice that

$$
\left|z^{\prime}-x\right| \leq\left|z^{\prime}-z\right|+|z-x| \leq 2 \varepsilon r+r+r_{Q} \leq 3 r .
$$

So $B_{P} \cap B(x, 3 r) \neq \varnothing$. Also, $y \in 10 B_{P}$ because

$$
\left|y-z^{\prime}\right| \leq|y-z|+\left|z-z^{\prime}\right| \leq r_{Q}+2 \varepsilon r \leq r_{Q}+2 \varepsilon^{1 / 2} r_{Q} \leq 2 r_{P},
$$

recalling (2.21). Hence, from (2.27) we infer that there exists $y^{\prime} \in 10 B_{P} \cap \partial \Omega_{\varepsilon}^{+}$such that $\left|y-y^{\prime}\right| \lesssim \varepsilon^{1 / 2} r$. So the proof of (2.19) is concluded.

The condition (b) in Definition 1.1 can be shown as in case 1, we omit the details.

Case 3. Suppose that $r \leq r_{Q}$ for some $Q \in \mathcal{I}$ such that $B_{Q} \cap B(x, 2 r) \neq \varnothing$.

In this case $B(x, r) \subset 10 B_{Q}$. Recalling that $\partial \Omega_{\varepsilon}^{+}$coincides on $10 B_{Q}$ with the graph of a Lipschitz function $f_{Q}: L_{Q} \rightarrow L_{Q}^{\perp}$ with Lipschitz constant not exceeding $c \varepsilon^{1 / 2}$, if we denote by $L_{Q, x}$ the hyperplane that is parallel to $L_{Q}$ and passes through $x$, we get by Lemmas 2.3 and 2.4

$$
\operatorname{dist}_{H}\left(\partial \Omega_{\varepsilon}^{+} \cap B(x, r), L_{Q, x} \cap B(x, r)\right) \lesssim \varepsilon^{1 / 2} r .
$$

Again, condition (b) in Definition 1.1 can be shown as in case 1, we omit the details. 


\section{RADON MEASURES OF LOW DIMENSION}

Some of our work toward the main result can be done in more generality than with harmonic measure. We will apply the following theorem in the last section with $\mu$ equal to the harmonic measure for a suitable modification of a Wolff snowflake domain.

Theorem 3.1. Let $\Omega$ be a $\left(\delta, r_{0}\right)$-Reifenberg flat domain, $\xi_{0} \in \partial \Omega$, and $E \subset$ $B\left(\xi_{0}, r_{0}\right) \cap \partial \Omega$ a closed set. Also assume that there is a Radon measure $\mu$ with support contained in $\partial \Omega$ such that $\mu(B(\xi, r)) \geq c_{\mu} r^{d-\alpha}$ for all $\xi \in E, r<r_{0}$ and some constants $c_{\mu}, \alpha>0$. If $\Omega_{\varepsilon}^{+} \supset \Omega$ is the domain from Lemma 2.2, then $\left.\mathcal{H}^{d}\right|_{\partial \Omega_{\varepsilon}^{+}}$ is locally finite.

Proof. For $Q \in \mathcal{I}$, let $\Gamma_{Q}=10 B_{Q} \cap \partial \Omega_{\varepsilon}^{+}$, so by Lemma 2.3, this is a Lipschitz graph.

Let $\xi \in \partial \Omega_{\varepsilon}^{+}$, and $r>0$ be much smaller than $r_{0}$ (how small will depend on $\varepsilon$ and $d$ ), and define

$$
C(\xi, r)=\left\{Q \in \mathcal{I}: \Gamma_{Q} \cap B(\xi, r) \neq \emptyset\right\} .
$$

Our goal now is to show that $\mathcal{H}^{d}\left(\partial \Omega_{\varepsilon}^{+} \cap B(\xi, r)\right)<\infty$ for all $r \in\left(0, r_{0}\right)$. We consider two cases.

Case 1. If $\operatorname{dist}(\xi, E) \geq 2 r$, then all cubes $Q \in C(\xi, r)$ have comparable sidelengths. To see this, suppose we can find $Q_{j} \in C(\xi, r)$ so that $\ell\left(Q_{j}\right) \rightarrow 0$. Then eventually, $\ell(Q) \ll r_{0}$ so that $\ell\left(Q_{j}\right) \sim \varepsilon^{2} \operatorname{dist}(x, E)$, thus

$$
2 r \leq \operatorname{dist}(\xi, E) \leq \liminf \left(\operatorname{dist}\left(\xi, Q_{j}\right)+\operatorname{diam} Q_{j}\right) \leq r+0,
$$

which gives a contradiction. Thus, $\inf \{\ell(Q): Q \in C(\xi, r)\}>0$. On the other hand, for all $Q \in C(\xi, r)$,

$$
\ell(Q) \lesssim \varepsilon^{2} \operatorname{dist}(Q, E) \leq \varepsilon^{2}(r+\operatorname{dist}(\xi, E))<\infty .
$$

Hence, all $Q \in C(\xi, r)$ have comparable diameters. Since they all intersect $B(\xi, r)$, this means there must be finitely many of them, and thus $\mathcal{H}^{d}\left(B(\xi, r) \cap \partial \Omega_{\varepsilon^{+}}\right)<\infty$.

Case 2. Now suppose $\operatorname{dist}(\xi, E)<2 r$. Note that

$$
\begin{aligned}
\operatorname{dist}(Q, E) & \lesssim \operatorname{dist}\left(Q, \Gamma_{Q}\right)+\operatorname{diam} \Gamma_{Q}+\operatorname{dist}\left(\Gamma_{Q}, \xi\right)+\operatorname{dist}(\xi, E) \\
& \lesssim r_{Q}+r+2 r \lesssim \varepsilon^{-1} \ell(Q) \lesssim \varepsilon \operatorname{dist}(Q, E)+r
\end{aligned}
$$

and so for $\varepsilon$ small enough $\operatorname{dist}(Q, E) \lesssim r$, and so $\operatorname{dist}(Q, E)<r_{0}$ if $r \ll r_{0}$, thus

$$
\ell(Q) \sim \varepsilon^{2} \operatorname{dist}(Q, E) \lesssim \varepsilon^{2} r
$$

for all $Q \in C(\xi, r)$. For $Q \in C(\xi, r)$, pick $\xi_{Q} \in E$ so that $\operatorname{dist}\left(\xi_{Q}, Q\right)=\operatorname{dist}(Q, E) \sim$ $\varepsilon^{-2} \ell(Q)$ and let $B^{Q}=B\left(\xi_{Q}, \ell(Q)\right)$. For $n \in \mathbb{Z}$, define

$$
C_{n}(\xi, r)=\left\{Q \in C(\xi, r): \ell(Q)=2^{-n}\right\} .
$$

We claim that there is $N_{1}=N_{1}(\varepsilon, d)$ so that no point in $E$ is contained in more than $N_{1}$ many $B^{Q}$ with $Q \in C_{n}(\xi, r)$. Thus, fix $n \in \mathbb{Z}$ and $\zeta \in E$. If $Q \in C_{n}(\xi, r)$ is 
such that $\zeta \in B^{Q}$, then

$$
\operatorname{dist}(\zeta, Q) \leq\left|\zeta-\xi_{Q}\right|+\operatorname{dist}\left(\xi_{Q}, Q\right)<c \ell(Q)+\operatorname{dist}(Q, E) \sim \varepsilon^{-2} \ell(Q)=\varepsilon^{-2} 2^{-n} .
$$

Thus, all cubes $Q \in C_{n}(\xi, r)$ for which $\zeta \in B^{Q}$ are disjoint, contained in a ball of radius $C 2^{-n}$ for some $C=C(\varepsilon, d)$, and are of side length $2^{-n}$, so there can only be at most $N_{1}=N_{1}(\varepsilon, d)$ many of them, which settles the claim.

Since $\ell(Q) \lesssim \varepsilon^{2} r$, and again for $r \ll r_{0}, \ell(Q)<r<r_{0}$. In particular, $C_{n}(\xi, r) \neq \emptyset$ implies $2^{-n} \leq r$. Thus, since $\operatorname{diam} \Gamma_{Q} \sim r_{Q} \sim \varepsilon^{-1} \ell(Q)$,

$$
\begin{aligned}
\left|\xi_{Q}-\xi\right| & \leq \operatorname{dist}\left(\xi_{Q}, Q\right)+\operatorname{diam} Q+\operatorname{dist}\left(Q, \Gamma_{Q}\right)+\operatorname{diam} \Gamma_{Q}+\operatorname{dist}\left(\xi, \Gamma_{Q}\right) \\
& \lesssim{ }_{d} \varepsilon^{-2} \ell(Q)+\ell(Q)+\varepsilon^{-1} \ell(Q)+\varepsilon^{-1} \ell(Q)+r \lesssim r .
\end{aligned}
$$

Thus, $Q \in C(\xi, r)$ implies $\xi_{Q} \in B(\xi,(C-1) r)$ for some large $C>0$, depending on $d$, and $\ell(Q)<r$ implies $B^{Q} \subset B(\xi, C r)$. Finally, note that $Q \in C_{n}(\xi, r)$ implies

$$
\mathcal{H}^{d}\left(\Gamma_{Q}\right) \sim \varepsilon^{-d} \ell(Q)^{d} \lesssim_{\varepsilon, d} \ell(Q)^{\alpha} r\left(B^{Q}\right)^{d-\alpha} \leq c_{\mu}^{-1} 2^{-n \alpha} \mu\left(B^{Q}\right) .
$$

Therefore,

$$
\begin{aligned}
\mathcal{H}^{d}\left(\partial \Omega_{\varepsilon}^{+} \cap B(\xi, r)\right) & \leq \sum_{Q \in C(\xi, r)} \mathcal{H}^{d}\left(\Gamma_{Q}\right)+\mathcal{H}^{d}(E)=\sum_{2^{-n} \leq r} \sum_{Q \in C_{n}(\xi, r)} \mathcal{H}^{d}\left(\Gamma_{Q}\right)+0 \\
& \lesssim \sum_{2^{-n} \leq r} \sum_{Q \in C_{n}(\xi, r)} \frac{\mu\left(B^{Q}\right)}{2^{n \alpha} c_{\mu}} \leq \frac{N_{1}}{c_{\mu}} \sum_{2^{-n} \leq r} 2^{-n \alpha} \mu(B(\xi, C r)) \\
& \lesssim \varepsilon, d, c_{\mu} r^{\alpha} \mu(B(\xi, C r))<\infty
\end{aligned}
$$

The proof of the theorem is finished now that we have shown these two cases.

\section{WolfF SNOWflakes AND harmonic MEASURE}

We will now describe the construction of the Wolff snowflake domain. We follow closely the approach of [LNV], which in turn is just a small variant of the original construction of Wolff in [W]. We also remark that the below description of the construction of the Wolff snowflakes is an almost verbatim copy of an analogous one in [LNV]. For more details, see the aforementioned references.

Let $\Omega_{0}=\left\{\left(x^{\prime}, x_{d+1}\right): x^{\prime} \in \mathbb{R}^{d}, x_{d+1}>0\right\}$ and set $Q(r):=\left\{x^{\prime} \in \mathbb{R}^{d}:-r / 2 \leq x_{i} \leq\right.$ $r / 2$, for $1 \leq i \leq d\}$. Then $Q(r)$ is a $d$-dimensional cube with side length $r$ and center 0 . Let $\phi: \mathbb{R}^{d} \rightarrow \mathbb{R}$ be a piecewise linear function with $\operatorname{supp}(\phi) \subset\left\{x^{\prime} \in \mathbb{R}^{d}:\left|x^{\prime}\right|<1 / 2\right.$ and $\|\nabla \phi\|_{\infty} \leq \theta$. For fixed $N$ large, set $\psi\left(x^{\prime}\right)=N^{-1} \phi\left(N x^{\prime}\right)$. Let $b>0$ be a small constant and let $Q$ be a $d$-cube (i.e., a $d$-dimensional cube contained in some hyperplane) with center $a_{Q}$ and side length $\ell(Q)$. Let $e$ be a unit normal to $Q$ and define

$$
P_{Q}=\operatorname{cch}\left(Q \cup\left\{a_{Q}+b \ell(Q) e\right\}\right), \quad \widetilde{P}_{Q}=\operatorname{int} \operatorname{cch}\left(Q \cup\left\{a_{Q}-b \ell(Q) e\right\}\right),
$$


where $\operatorname{cch} E$ and $\operatorname{int} E$ denote the closed convex hull and interior of $E$, respectively. For the cube $Q(1)$ set $e=-e_{n}=(0, \ldots, 0,-1)$ and let

$$
\begin{gathered}
\Lambda=\left\{x \in P_{Q(1)} \cup \widetilde{P}_{Q(1)}: x_{d+1}>\psi(x)\right\} \\
\partial=\left\{x \in \mathbb{R}^{d+1}: x^{\prime} \in Q(1), x_{d+1}=\psi\left(x^{\prime}\right)\right\} .
\end{gathered}
$$

We assume that $N=N(b, \theta)$ is so large that $\operatorname{dist}\left(\partial \backslash \partial \Omega_{0}, \partial\left[P_{Q(1)} \cup \widetilde{P}_{Q(1)}\right]\right) \geq$ $b / 100$. Note that $\partial \subset Q(1) \times[-1 / 2,1 / 2]$ consists of a finite number of $d$-dimensional faces. We fix a Whitney decomposition of each face. That is, we divide each face of $\partial$ into $d$-cubes $Q$, with side lengths $8^{-k}, k=1,2, \ldots$ which are proportional to their distance from the edges of the face they lie on. We also choose a distinguished $(d-1)$-dimensional "side" for each $d$-cube.

Suppose $\Omega$ is a domain and $Q \subset \partial \Omega$ is a $d$-cube with distinguished side $\gamma$. Let $e$ be the outer unit normal to $\partial \Omega$ on $Q$ and suppose that $P_{Q} \cap \Omega=\emptyset$ and $\widetilde{P}_{Q} \subset \Omega$. We form a new domain $\widetilde{\Omega}$ as follows. Let $T$ be the conformal affine map (i.e., a composition of a translation, rotation, dilation) with $T(Q(1))=Q$ which fixes the dilation, $T(0)=a_{Q}$ which fixes the translation, and finally fix the rotation by requiring that $T\left(\left\{x \in \partial Q(1): x_{1}=1 / 2\right\}\right)=\gamma$ and $T\left(-e_{n}\right)$ is in the direction of $e$. Let $\Lambda_{Q}=T(\Lambda)$ and $\partial_{Q}=T(\partial)$. Then we define $\widetilde{\Omega}$ through the relations $\widetilde{\Omega} \cap\left(P_{Q} \cup \widetilde{P}_{Q}\right)=\Lambda_{Q}$ and $\widetilde{\Omega} \backslash\left(P_{Q} \cup \widetilde{P}_{Q}\right)=\Omega \backslash\left(P_{Q} \cup \widetilde{P}_{Q}\right)$. Note that $\partial_{Q}$ inherits from $\partial$ a natural subdivision into Whitney cubes with distinguished sides. We call this process "adding a blip to $\Omega$ along $Q$ ".

To use the process of "adding a blip" to construct a Wolff snowflake $\Omega_{\infty}$, starting from $\Omega_{0}$, we first add a blip to $\Omega_{0}$ along $Q(1)$ obtaining a new domain $\Omega_{1}$. We then inherit a subdivision of $\partial \Omega_{1} \cap\left(P_{Q(1)} \cup \widetilde{P}_{Q(1)}\right)$ into Whitney cubes with distinguished sides, together with a finite set of edges $E_{1}$ (the edges of the faces of the graph are not in the Whitney cubes). Let $G_{1}$ be the set of all Whitney cubes in the subdivision. Then $\Omega_{2}$ is obtained from $\Omega_{1}$ by adding a blip along each $Q \in G_{1}$. From this process, we inherit a family of cubes $G_{2} \subset \partial \Omega_{2}$ (each with a distinguished side) and a set of edges $E_{2} \subset \partial \Omega_{2}$ of $\sigma$-finite $\mathcal{H}^{d-1}$-measure. Continuing by induction we get $\left(\Omega_{m}\right)_{m=1}^{\infty}$, $\left(G_{m}\right)_{m=1}^{\infty}$ and $\left(E_{m}\right)_{m=1}^{\infty}$, where $\partial \Omega_{m} \cap\left(P_{Q(1)} \cup \widetilde{P}_{Q(1)}\right)=E_{m} \cup \bigcup_{Q \in G_{m}} Q$ for $m \geq 1$. If $N=N(b, \theta)$ is large enough, then $\operatorname{dist}_{H}\left(\Omega_{m}, \Omega_{\infty}\right) \rightarrow 0$ as $m \rightarrow \infty$. We call $\Omega_{\infty}$ a $\theta$-Wolff snowflake domain.

The following result is proved in [LNV, Lemma 7.1].

Lemma 4.1. Suppose that $\theta \in(0,1)$ is small enough and $N$ large enough, depending on $d, b$, and $\theta$. Then the $\theta$-Wolff snowflake domain $\Omega_{\infty}$ is $\left(c_{1} \theta, \infty\right)$-Reifenberg flat, for some positive constant $c_{1}$.

Remark 4.2. Similarly, for any fixed $\varepsilon>0$ and $\tau>0$, one may construct a bounded $\theta$-Wolff snowflake domain (see also $[\mathrm{LNV}]$ and $[\mathrm{W}]$ ). Indeed, this is done by taking the unit cube in $\mathbb{R}^{d+1}$ contained in the lower half-space that has $Q(1)$ as one of its faces (its "bottom" face). Then we just mimic the construction above to each face of the cube. We will denote this new domain by $\widetilde{\Omega}_{\infty}$. Notice here that $\widetilde{\Omega}_{\infty} \subset \Omega_{\infty}$ 
and

$$
\partial \widetilde{\Omega}_{\infty} \cap\left\{x \in \mathbb{R}^{d+1}: x_{d+1}<0\right\}=\partial \Omega_{\infty} \cap\left\{x \in \mathbb{R}^{d+1}: x_{d+1}<0\right\} .
$$

Before we apply our results from the previous section let us introduce some notation. If $\mu$ is a Borel probability measure in $\mathbb{R}^{d+1}$, we define its lower pointwise dimension at the point $x \in \operatorname{supp} \mu$ to be

$$
\underline{d}_{\mu}(x)=\liminf _{r \rightarrow 0} \frac{\log \mu(B(x, r))}{\log r}
$$

and its upper pointwise dimension at the point $x \in \operatorname{supp} \mu$

$$
\bar{d}_{\mu}(x)=\limsup _{r \rightarrow 0} \frac{\log \mu(B(x, r))}{\log r} .
$$

The common value $\underline{d}_{\mu}(x)=\bar{d}_{\mu}(x)=d_{\mu}(x)$, if it exists, we call it pointwise dimension of $\mu$ at $x \in \operatorname{supp} \mu$.

Let $\operatorname{dim}_{H}(Z)$ be the Hausdorff dimension of the set $Z$. Given a measure $\mu$ on a set $\Lambda \subset \mathbb{R}^{d+1}$ the Hausdorff dimension of $\mu$ is defined by

$$
\operatorname{dim}_{H}(\mu)=\inf \left\{\operatorname{dim}_{H}(Z): Z \subset \Lambda \text { and } \mu(\Lambda \backslash Z)=0\right\} .
$$

Moreover,

$$
\operatorname{dim}_{H}(\mu)=\operatorname{ess} \sup \left\{\underline{d}_{\mu}(x): x \in \Lambda\right\}
$$

where the essential supremum is taken with respect to $\mu$ (see Proposition 3, [BW]). In particular, if there exists a number $\delta$ so that $d_{\mu}(x)=\delta$ for $\mu$-a.e. $x \in \Lambda$, then $\operatorname{dim}_{H}(\mu)=\delta$. This criterion was established in [Y] by Young.

The following theorem was proved by Wolff $[\mathrm{W}]$

Theorem 4.3. For every $\theta>0$ there exists a bounded $\theta$-Wolff snowflake domain $\widetilde{\Omega}_{\infty} \subset \mathbb{R}^{d+1}$ built from a Lipschitz function function $\phi$ with Lipschitz constant at most $\theta$ such that

$$
d_{\omega_{\widetilde{\Omega}_{\infty}}}(x)=s<d, \quad \text { for all } x \in \partial \widetilde{\Omega}_{\infty} \backslash \mathcal{X},
$$

where $\mathcal{X} \subset \widetilde{\Omega}_{\infty}$ is such that $\omega_{\widetilde{\Omega}_{\infty}}(\mathcal{X})=0$ and $\omega_{\widetilde{\Omega}_{\infty}}$ is the harmonic measure in $\widetilde{\Omega}_{\infty}$.

We shall use now Lemma 4.1 and Theorem 4.3 in order to apply the results from the previous sections and obtain our main theorem.

Note that from now we identify $\left\{\left(\tilde{x}, x_{d+1}\right) \in \mathbb{R}^{d+1}: x_{d+1}=0\right\}$ with $\mathbb{R}^{d}$.

Proof of Theorem 1.2. Let $\theta>0$ be a sufficiently small constant that will be chosen momentarily and assume that $\widetilde{\Omega}_{\infty}$ is a $\theta$-Wolff snowflake domain. Let us also fix a pole $z_{0} \in \widetilde{\Omega}_{\infty}$.

By Theorem 4.3 we have that $d_{\omega_{\widetilde{\Omega}_{\infty}}}(\xi) \leq s<d$ for $\omega_{\widetilde{\Omega}_{\infty}}$-a.e. $\xi \in \partial \widetilde{\Omega}_{\infty}$ and thus, $\operatorname{dim}_{H}\left(\omega_{\widetilde{\Omega}_{\infty}}\right) \leq s<d$. This implies that there exists a set $X \subset \partial \widetilde{\Omega}_{\infty}$ so that $\omega_{\widetilde{\Omega}_{\infty}}(X)=1, \operatorname{dim}_{H}(X) \leq s<d$ (which implies $\mathcal{H}^{d}(X)=0$ ) and $d_{\omega_{\widetilde{\Omega}_{\infty}}}(\xi) \leq s<d$ for every $\xi \in X$. Therefore, if we set

$$
Z_{0}:=\left\{\xi \in X \cap \partial \Omega_{\infty} \backslash\left(\mathbb{R}^{d} \backslash Q(1)\right): d_{\omega_{\tilde{\Omega}_{\infty}}}(\xi) \leq s\right\},
$$


then $\omega_{\widetilde{\Omega}_{\infty}}\left(Z_{0}\right)>0$ (recall that by the earlier remark, $\left.\partial \Omega_{\infty} \backslash\left(\mathbb{R}^{d} \cup Q(1)\right) \subset \partial \tilde{\Omega}_{\infty}\right)$. Furthermore, for $\alpha \in(0, d-s)$, there exists $\rho_{0} \leq \min \left\{\operatorname{diam} \widetilde{\Omega}_{\infty}, 1\right\}$ such that $\omega_{\widetilde{\Omega}_{\infty}}\left(Z_{1}\right)>0$, where

$$
Z_{1}:=\left\{\xi \in Z_{0}: \frac{\log \omega_{\widetilde{\Omega}_{\infty}}\left(B(\xi, r) \cap \partial \widetilde{\Omega}_{\infty}\right)}{\log r}<d-\alpha, \text { for any } r \in\left(0, \rho_{0}\right]\right\} .
$$

Notice that this implies that $\omega_{\widetilde{\Omega}_{\infty}}\left(B(\xi, r) \cap \partial \widetilde{\Omega}_{\infty}\right)>r^{d-\alpha}$ for any $\xi \in Z_{1}$ and $0<r \leq \rho_{0} \leq 1$.

Let us fix $\xi_{0} \in Z_{1}$. By the inner regularity of $\omega_{\widetilde{\Omega}_{\infty}}$, there exist $r_{0} \in\left(0, \rho_{0}\right]$ and a compact set $E \subset Z_{1} \cap B\left(\xi_{0}, r_{0}\right)$ so that $\omega_{\widetilde{\Omega}_{\infty}}(E)>0$ and $\omega_{\widetilde{\Omega}_{\infty}}\left(B(\xi, r) \cap \partial \widetilde{\Omega}_{\infty}\right)>r^{d-\alpha}$ for every $\xi \in E$ and $r \in\left(0, r_{0}\right)$. Since $E \subset \partial \widetilde{\Omega}_{\infty} \cap \partial \Omega_{\infty}$ and $\widetilde{\widetilde{\Omega}}_{\infty} \subset \bar{\Omega}_{\infty}$, in view of the maximum principle, we have that $\omega_{\Omega_{\infty}}(E)>0$ and $\omega_{\Omega_{\infty}}\left(B(\xi, r) \cap \partial \Omega_{\infty}\right)>r^{d-\alpha}$ for every $\xi \in E$ and $r \in\left(0, r_{0}\right)$.

We now fix $\varepsilon>0$ small enough. By Lemmas 2.2 and 4.1, if we choose $\theta \in(0,1)$ so that $c_{1} \theta \leq \delta_{0}$, taking into account that $\Omega_{\infty}$ is $\left(c_{1} \theta, \infty\right)$-Reifenberg flat and hence $\left(\delta_{0}, \infty\right)$-Reifenberg flat, we infer that there exists a $\left(c \varepsilon^{1 / 2}, \infty\right)$-Reifenberg flat domain $\Omega$ such that $\bar{\Omega}_{\infty} \subset \bar{\Omega}$ and $E \subset \partial \Omega \cap \partial \Omega_{\infty}$. Once again, we apply the maximum principle and have that $\omega_{\Omega}(E)>0$ and $\omega_{\Omega}\left(B(\xi, r) \cap \partial \Omega_{\infty}\right)>r^{d-\alpha}$ for every $\xi \in E$ and $r \in\left(0, r_{0}\right)$. Hence, by Theorem 3.1 we obtain that $\left.\mathcal{H}^{d}\right|_{\partial \Omega}$ is locally finite, that is, Radon. This concludes our theorem since $E$ is a compact subset of $\Omega$ so that $\mathcal{H}^{d}(E)=0$ and $\omega_{\Omega}(E)>0$.

\section{REFERENCES}

[AHMNT] J. Azzam, S. Hofmann, J.M. Martell, K. Nyström and T. Toro, A new characterization of chord-arc domains, arXiv:1406.2743 [math.CA]. To appear in J. Eur. Math. Soc. (JEMS). 2

[AT] J. Azzam and X. Tolsa, Characterization of n-rectifiability in terms of Jones' square function: Part II. Geom. Funct. Anal. 25 (2015), no. 5, 1371-1412. 10

[Ba] M. Badger, Null sets of harmonic measure on NTA domains: Lipschitz approximation revisited, Math. Z. 270 (2012), no. 1-2, 241-262. MR 2875832 (2012k:31008) 2

[BW] L. Barreira and C. Wolf, Pointwise dimension and ergodic decompositions. Ergodic Theory Dynam. Systems 26 (2006), 653-671. 16

[BL] B. Bennewitz and J. Lewis, On weak reverse Hölder inequalities for nondoubling harmonic measures, Complex Var. Theory Appl. 49 (2004), no. 7-9, 571-582. 2

[Da] G. David, Approximation of a Reifenberg-flat set by a smooth surface, Bull. Belg. Math. Soc. Simon Stevin 21 (2014), no. 2, 319-338. 3

[DJ] G. David and D. Jerison, Lipschitz approximation to hypersurfaces, harmonic measure, and singular integrals, Indiana Univ. Math. J. 39 (1990), no. 3, 831-845. MR 1078740 (92b:42021) 2

[HMU] S. Hofmann, J.M. Martell, I. Uriarte-Tuero, Uniform rectifiability and harmonic measure, II: Poisson kernels in $L^{p}$ imply uniform rectifiability, Duke Math. J. 163 (2014), no. 8, 1601?-1654. 2

[KPT] C. Kenig, D. Preiss and T. Toro, Boundary structure and size in terms of interior and exterior harmonic measures in higher dimensions, J. Amer. Math. Soc. 22 (2009), no. 3, 771-796. 2 
[KT] C. E. Kenig and T. Toro, Harmonic measure on locally flat domains. Duke Math. J. 87 (1997), no. 3, 509-551. 2, 4

[LMS] A. Lemenant, E. Milakis and L. Spinolo, On the extension property of Reifenberg-flat domains. Ann. Acad. Sci. Fenn. Math. 39 (2014), no. 1, 51-71. 2

[LNV] J. L. Lewis, K. Nyström and A. Vogel, On the dimension of p-harmonic measure in space. J. Eur. Math. Soc. 15, 2197-2256. 14, 15

[LVV] J. L. Lewis, G. C. Verchota, and A. L. Vogel, Wolff snowflakes, Pacific J. Math. 218 (2005), no. 1, 139-166. MR 2224593 (2006m:31005) 3

[W] T. Wolff, Counterexamples with harmonic gradients in $\mathbb{R}^{3}$. In: Essays on Fourier Analysis in Honor of Elias M. Stein, Princeton Math. Ser. 42, Princeton Univ. Press, 321-384 (1995). 3, 14, 15, 16

[Wu J-M. Wu, On singularity of harmonic measure in space, Pacific J. Math. 121 (1986), no. 2, 485-496. MR 819202 (87e:31009) 1

[Y] L.-S. Young, Dimension, entropy and Lyapunov exponents. Ergodic Theory Dynam. Systems 2, 109-124 (1982). 16

[Z] W. Ziemer, Some remarks on harmonic measure in space, Pacific J. Math. 55 (1974), no. 2, 629-637. 1

Departament de Matemàtiques, Universitat Autònoma de Barcelona, Edifici C Facultat de CiÈncies, 08193 Bellaterra (Barcelona, Catalonia)

E-mail address: jazzam@mat.uab.cat

Departament de Matemàtiques, Universitat Autònoma de Barcelona and Centre de Reserca Matemàtica, Edifici C Facultat de Ciències, 08193 Bellaterra (Barcelona, Catalonia)

E-mail address: mmourgoglou@crm.cat

iCREA and Departament de Matemàtiques, Universitat Autònoma de Barcelona, Edifici C Facultat de Ciències, 08193 Bellaterra (Barcelona, Catalonia)

E-mail address: xtolsa@mat.uab.cat 